\title{
Essais
}

Revue interdisciplinaire d'Humanités

$12 \mid 2017$

Textes et contextes : entre autonomie et dépendance

\section{Texte, cotexte, contexte à la lumière de l'hypothèse saussurienne des anagrammes}

Federico Bravo

\section{(2) OpenEdition}

1 Journals

Édition électronique

URL : http://journals.openedition.org/essais/2832

DOI : $10.4000 /$ essais. 2832

ISSN : 2276-0970

Éditeur

École doctorale Montaigne Humanités

Édition imprimée

Date de publication : 15 juin 2017

Pagination : 89-99

ISBN : 979-10-97024-02-4

ISSN : 2417-4211

Référence électronique

Federico Bravo, «Texte, cotexte, contexte à la lumière de l'hypothèse saussurienne des

anagrammes », Essais [En ligne], 12 | 2017, mis en ligne le 12 octobre 2020, consulté le 25 octobre

2020. URL : http://journals.openedition.org/essais/2832 ; DOI : https://doi.org/10.4000/essais.2832 


\title{
Texte, cotexte, contexte à la lumière de l'hypothèse saussurienne des anagrammes
}

\author{
Federico Bravo
}

L'aptitude migratoire des unités textuelles dont se sustente le dispositif paragrammatique imaginé par Ferdinand de Saussure dans ses Cahiers d'anagrammes relève d'une conception tectonique du texte à la croisée de l'oral et de l'écrit. Délinéarisé, déconstruit, tabularisé, le texte devient surface mouvante, en trompe l'œil. À la fois contenu latent (hypotexte) et antécédent du texte (prétexte), l'anagramme invite à s'interroger sur l'articulation du binôme texte/contexte, à réfléchir à l'irréductible du texte et à repenser la textualité comme une forme de dépassement du clivage oralité/scripturalité.

Tel que le conçoit Saussure (c'est ainsi, au masculin, que le linguiste emploie le terme), l'anagramme n'est pas une figure du mot, mais une figure $\mathrm{du}$ discours tout entier. En cela il met en cause une conception singulière du texte ${ }^{1}$. L'anagramme pour Saussure est bien plus qu'une structure onomastique ensevelie dans un ensemble de vers, un nom propre caché dans le texte. Il s'agit d'une structure générative au sens où c'est le signifiant onomastique qui génère le texte et qui guide l'écriture : l'anagramme saussurien n'est pas un jeu ou un artifice mais une "manière d'être " du texte, qui doit être saisi dans son fonctionnement " séminal ${ }^{2}$ ", c'est-à-dire dans son aptitude à générer

1 En effet, loin d'être confinée au décryptage de quelques signifiants onomastiques, la théorie de l'anagramme non seulement se fonde sur une théorie du texte, mais se construit elle-même comme une théorie du texte. S'il est vrai qu'en découvrant le principe ordonnant des hymnes védiques, du vers Saturnien, de la poésie germanique allitérante, Ferdinand de Saussure a contribué de manière décisive au dégagement et à la reconstruction d'une poétique indo-européenne, l'intérêt de la théorie de l'anagramme réside, autant que dans la découverte elle-même, dans ce que cette découverte a permis de comprendre sur les mécanismes psycholinguistiques qui sont à l'œuvre dans le processus de création.

2 Starobinski Jean, Les mots sous les mots. Les anagrammes de Ferdinand de Saussure, Paris, Gallimard, 1971, p. 61 (rééd. Lambert-Lucas, Limoges, 2009). Mentionnons également Wunderli Peter, Ferdinand de Saussure und die Anagramme, Linguistik und Literatur, Tübingen, Max Niemeyer Verlag, 1972, parmi les pionniers. Cf. l'étude incontournable de Francis Gandon, De dangerenx édifices. Saussure lecteur de Lucrèce. Les cahiers d'anagrammes consacrés au "De rerum 
ou à laisser lire ou entendre d'autres co-textes. En effet, ce que Saussure a mis en lumière en signalant la présence de structures onomastiques plus ou moins clandestines à l'intérieur des vers saturniens ${ }^{3}$ c'est surtout, comme l'a justement souligné Thomas Aron, " un fonctionnement autre du texte, et du langage dans le texte ${ }^{4}$ ". La notion on ne peut plus diffuse d'inspiration (ce qu'on appelle le souffle créateur, la muse, la source de l'écriture poétique, la créativité, etc.) trouve ainsi dans le nom propre une expression visible et matérielle, puisque c'est la configuration littérale du signifiant onomastique qui sert de base à la création et qui consciemment ou inconsciemment guide le travail du créateur. Saussure parle de mot-thème pour désigner le nom que le texte, sa paraphrase phonique, devait imiter, le poème se construisant comme une série de variations au sens musical du terme autour du même thème onomastique :

La méthode habituelle et fondamentale du poète consistait à décomposer préalablement le mot-thème, et à s'inspirer de ses syllabes pour les idées qu'il allait émettre ou les expressions qu'il allait choisir. C'est sur les morceaux de l'anagramme, pris comme cadre et comme base, qu'on commençait le travail de composition. ${ }^{5}$

Quelque subjectivité qu'on lui suppose, l'anagramme est loin d'être une pratique déréglementée 6 . Il ne m’est pas permis dans le cadre du présent travail

natura ", Louvain-Paris, Éditions Peeters, 2002. Nous avons abordé ces questions dans "En relisant les anagrammes de Saussure. Pour une sémiologie des "figures sonores" ", Théories $d u$ texte et pratiques méthodologiques, Presses Universitaires de Caen, 2000, p. 55-68 ; "Polémico Saussure: la hipótesis del anagrama o los albores de un escándalo estructural ", Despalabro. Ensayos de humanidades, Universidad Autónoma de Madrid, n 6, 2012, p. 27-44; Anagrammes. Sur une hypothèse de Ferdinand de Saussure, Limoges, Lambert-Lucas, 2011.

3 Les recherches de Saussure sur le Saturnien - ce vers énigmatique qui n'obéit à aucun schéma connu de la métrique classique et dont il croit être enfin parvenu à percer le mystère - sont à l'origine de la spéculation autour de l'anagramme. Dans une lettre datée du 14 juillet 1906, après plusieurs mois de recherches consacrés à l'étude du Saturnien, il écrit dans un état de fébrilité : « ... je puis vous annoncer que je tiens maintenant la victoire sur toute la ligne. J'ai passé deux mois à interroger le monstre, et à n'opérer qu'à tâtons contre lui, mais depuis trois jours je ne marche plus qu'à coups de grosse artillerie [...] c'est par l'Allitération que je suis arrivé à tenir la clef du Saturnien autrement compliqué qu'on ne se le figurait. Tout le phénomène de l'allitération [...] qu'on remarquait dans le Saturnien, n'est qu'une insignifiante partie d'un phénomène plus général, ou plutôt absolument total. La totalité des syllabes de chaque vers Saturnien obéit à une loi d'allitération, de la première syllabe à la dernière; et sans qu'une seule consonne $[\ldots]$ une seule voyelle $[\ldots]$ une seule quantité de voyelle, ne soit scrupuleusement portée en compte. Le résultat est tellement surprenant qu'on est porté à se demander avant tout comment les auteurs de ces vers [...] pouvaient avoir le temps de se livrer à un pareil casse-tête : car c'est un véritable jeu chinois..." (Starobinski, 1971, p. 20-21).

4 Aron Thomas, «Une seconde révolution saussurienne », Langue française, $\mathrm{n}^{\circ}$ 7, 1970, p. 59-60.

5 Starobinski, ibid., p. 127.

6 En effet, réfléchir à l'anagramme en tant que procédé d'exploration du texte revient à s'interroger sur les conditions qui en légitiment l'utilisation au cours de l'analyse et sur l'acceptabilité des lectures cryptographiques ainsi mises au jour, question souvent évoquée par la critique postsaussurienne, mais largement escamotée aussi dans la plupart des études qui abordent le sujet. 
de passer en revue les règles complexes - et souvent changeantes - auxquelles obéit le principe de construction textuelle mis au jour par le linguiste. Je me contenterai de rappeler ici qu'une première restriction importante est celle qui émane du principe du diphone. Bien que l'anagramme soit né d'une valorisation à la fois fonctionnelle et esthétique des unités minimales de la chaîne parlée ou écrite, son unité de mesure n'est, le plus souvent et contrairement à ce que laisse penser sa définition courante, ni le phonème ni la lettre, mais une suite de deux, trois, voire quatre de ces unités. En effet la plupart des anagrammes mis en lumière par Saussure ne sont pas de simples combinaisons de phonèmes, mais des combinaisons de combinaisons de phonèmes. Les conséquences sur le plan de l'analyse d'un tel principe sont considérables, car les chances de trouver dans un texte des unités dont la récurrence soit statistiquement parlant significative s'amenuisent à mesure que l'on élargit l'unité recherchée à des complexes phoniques et que l'on passe de la recherche d'un monophone à celle d'un diphone, d'un triphone ou d'un tétraphone. L'anagramme Falerni que Saussure détecte sous les vers de Pascoli «FAcundi cALices hausERe [...] ALtERNI7 " est, peut-on dire, d'autant plus vraisemblable qu'il se présente sous forme de syllabogramme et que le mot alterni contient tout entier le tétraphone ERNI qui, à lui tout seul, " imite " et rappelle paronymiquement le mot-thème ${ }^{8}$.

Or, si du fait de cette paronymie, l'exemple convoqué en témoignage semble plausible, dans bien des cas, pour ne pas dire en permanence, Saussure permute les sons, fait des métathèses, inverse l'ordre des éléments, les déplace,

7 Pascoli Giovanni, Catullocalvos - Satura, 1897, in Carmina, Milano, Mondadori, 1980.

8 Le procédé, on le voit, n’est pas très différent de celui qu'adopte, plus ludiquement, Michel Leiris dans son Glossaire lorsqu’à mi-chemin entre le mot dévalisé et le dépeçage syllabogrammatique il disperse dans sa définition lexicographique les lambeaux sonores du signifiant mis en vedette : "sacrilège - un SAC de CRIs l'assiĖGE », "marécage - mariage de sec et d'eau ; pacage d'insectes ", " jouissance - jusqu'où (en jouant) se hissent mes sens? ». Il ne s'agit pas cependant de nier la légitimité de l'anagramme considéré dans sa manifestation minimale en tant que mot obtenu par permutation des lettres d'un autre mot, ni de minimiser l'intérêt des associations parfois heureuses (chien/niche, Marielaimer, image/magie, parisien/aspirine, guérison/ soigneur, etc.) auxquelles ce type de dérivation peut donner lieu, comme c'est le cas lorsque William Wordsworth, dans le sonnet qu'il dédie au fondateur d'Haïti, réussit la métamorphose du prénom Toussaint en exultations (TOUSSAINT, the most unhappy man of men! [...] Thy friends are exULTATIONS, agonies, / And love...), lorsque Giacomo Leopardi transforme le nom de Silvia en verbe salivi (SILVIA, rimembri ancoral quel tempo della tua vita mortale,/ quando [...] tu, lieta e pensosa, il limitarel di gioventù SALIVI ?) ou lorsqu'Antonio Machado fait dériver blanco de balcón (Del abierto BALCÓN al BLANCO muro), máquina de camina (El tren CAMINA y CAMINA,/ y la MÁQUINA resuella), violines de livianos (con LIVIANOS acordes de VIOLINES) ou tahúres de truhanes (fulleros y TRUHANES,/ caciques y TAHÚRES). Mais il s'agit là de jeux ponctuels ou d'élucubrations de l'esprit (ang. : eleven plus two = twelve plus one, Mary $=$ army, it. : travaglio $=$ giravolta $=$ volgarità, esp. $:$ anagrama $=$ amar gana, recluta $=$ cuartel $)$ dont la portée ne dépasse pas les limites du mot. Or, nous l'avons dit, l'anagramme saussurien n'est pas une figure du mot, mais bien une figure du discours tout entier. 
met en relief telle séquence ou ignore complètement telle autre, et c'est là qu'intervient crucialement le contexte. En effet, le texte apparaît comme le lieu de transactions multiples entre le mot et son contexte plus ou moins immédiat. Le mot contexte n'apparait qu'épisodiquement dans les cahiers : Saussure parle plutôt de l'environnement d'un mot, de son entourage, en prêtant à l'un les propriétés de l'autre comme si, finalement, toutes sortes de transfusions étaient possibles entre les mots du fait de leur proximité. Le texte construit étant porteur d'un autre texte à construire, le contexte qui en permet l'émergence fait l'objet d'une extrême valorisation puisque c'est lui finalement qui incite à lire ou à entendre tel nom plutôt que tel autre enchâssé dans une suite de vers. L'anagramme n'est pas une question de quantité mais de qualité du contexte phonique : c'est dans le contexte (ou, dans l'environnement textuel), que la lecture anagrammatique trouve son alibi. Pour faire bref, on dira que le texte saussurien est gouverné par trois principes complémentaires, qui sont autant de concevabilités du texte et de la chose écrite, à savoir : 1 . un principe de mouvance textuelle (la lettre fixe le texte mais, paradoxalement, le texte n'est pas fixé, puisqu'il "bouge " au sens où le lecteur le voit devenir un autre texte), 2 . un principe de saillance (le texte ne signifie pas toujours et partout de la même manière ni avec la même intensité, il y a des endroits stratégiques, ce qui permet de valoriser dans le texte certaines séquences au détriment d'autres n'exerçant à leur égard qu'une fonction de catalyse) et 3. un principe de prolifération (le texte se diffracte en une multitude de co-textes, il est littéralement tapissé d'anagrammes : "il n'y a qu’à se baisser pour en ramasser à foison »). Tabularisation et contextualisation sont les maîtres mots : tabularisation du texte et contextualisation de ces bribes de signifiant onomastique disséminées dans le texte. Le texte étant perçu comme une surface en mouvement à laquelle l'exercice de l'interprétation doit se rapporter sous peine de rater son objectif, lire consiste désormais à reconnaître l'aptitude migratoire des lettres et des phonèmes : un tel dispositif mérite pleinement le qualificatif de tectonique voire de " textonique » si on veut jouer avec les mots.

Sur le plan linguistique, le modus operandi mis en lumière par l'auteur contredit frontalement, on ne l'a que trop signalé, le principe de la linéarité du signe, principe que Saussure énonce sur le ton de la loi dans son Cours de linguistique générale (mais qu'il relativise, on le sait moins, dans ses Écrits'). La théorie de l'anagramme est, sans aucun doute, la plus anti-saussurienne des théories puisqu' elle ébranle, d'un même mouvement, et le principe de la linéarité et celui de l'arbitraire du signe. Il est vrai, comme le signale Michel Arrivé que l'on du mal "à imaginer Saussure écrivant le même jour, mais sur deux cahiers différents ", d'une part, pour son Cours de linguistique générale, "le signifiant [...] se déroule dans le temps [...et n'est] mesurable [que] dans une

9 Saussure Ferdinand de, Écrits de linguistique générale, Paris, Gallimard, 2002. 
seule dimension ", et, d'autre part, à propos du Saturnien, " [l'anagramme] invite le lecteur [...] à amalgamer [les syllabes] hors du temps comme [on pourrait] le faire pour deux couleurs simultanées ${ }^{10} »$. Il reste cependant que le problème que pose ou que semble poser l'anagramme, qui apparaît comme une sorte de "scandale structural ${ }^{11}$ ", n'est pas fondamentalement différent de celui que pose un procédé comme la rime dont le propre pourtant est, comme pour l'anagramme, de suspendre la temporalité chronologique du langage au bénéfice d'une temporalité analogique, l'anagramme n'étant techniquement autre chose qu'une rime disséminée dans le texte.

L'analogie entre les sons et les couleurs n'est pas anodine sous la plume de Saussure, qui met ainsi explicitement en concurrence vision et audition. En effet, les manuscrits laissés par Saussure parlent de "l'attachement à la lettre " comme du " premier principe de la poésie indo-européenne ${ }^{12}$ ", tout en promouvant les poètes au rang de "phonétistes » et de «techniciens exercés par leur métier à connaître un son d'un autre ${ }^{13}$ ». Et si l'auteur parle d'anagramme, de para-gramme ou de crypto-gramme, il parle aussi de di-phone, d'ana-phonie et de substance phoni-que. Face à ce va-et-vient incessant entre la lettre et le son, son commentateur Jean Starobinski ne tranche pas : «l'œil et l'oreille exercés - écrit-il - [font] leur butin jusque dans la prose latine ${ }^{14}$ ". Mais que faut-il entendre par " œil et oreille exercés " ? En un mot : que « lit " Saussure : des lettres ou des sons?

Qu'il soit saisi par les yeux ou qu'il soit identifié par les oreilles, l'anagramme impose, par le retour incessant des motifs destinés à être reconnus comme autant de fragments épars du signifiant ayant servi de thème musical à la composition, un rythme basé sur l'alternance de séquences saillantes et de séquences de catalyse et façonne le discours en instrument d'ordre et de mesure. Comme le rappelle Henri Meschonnic, l'approche rythmique du langage dissout les frontières entre oralité et scripturalité : «Il n'y a pas d'un côté, l'audition, sens du temps, d'un autre la vision, sens de l'espace. Le rythme

10 Arrivé Michel, Langage et psychanalyse, linguistique et inconscient (Freud, Saussure, Pichon, Lacan), Paris, PUF, 1994, p. 61. "Peut-on donner TAE par $t a+t e-s e$ demande Saussure c'est-à-dire inviter le lecteur non plus à une juxtaposition dans la consécutivité, mais à une moyenne des impressions acoustiques hors du temps ? hors de l'ordre dans le temps qu'ont les éléments? hors de l'ordre linéaire qui est observé si je donne TAE par TA - AE ou TA - E, mais ne l'est pas si je le donne par $t a+t e$ à amalgamer hors du temps comme je pourrais le faire pour deux couleurs simultanées" (Starobinski, op. cit., 1971, p. 47).

11 Shepheard David, "Saussure et la loi poétique ", Présence de Saussure. Actes du Colloque international de Genève (21-23 mars 1988), Genève, Droz, 1988, p. 244.

12 Starobinski, ibid., p. 38.

13 Benveniste Émile, "Lettres de Ferdinand de Saussure à Antoine Meillet ", Cahiers Ferdinand de Saussure, n 21, 1964, p. 114.

14 Starobinski, ibid., p. 116. 
met de la vision dans l'audition ${ }^{15}$ ». Fictivement désassujetti du temps qui " change toute chose ", l'anagramme fournit une représentation elle-même virtuelle et illusoire du signe, montré " en apesanteur ", mais tout entier recolligible dans un présent psychologique : si, même éclaté, démembré, diffracté, analysé, dissous, décomposé, dispersé et dépecé - pour reprendre quelquesuns des termes employés et déployés par Jean Baudrillard ${ }^{16}$, le nom de la divinité qui se cache dans le texte reste un signe reconnaissable, c'est que les lambeaux qui en font l'anamnèse sont d'un point de vue cognitif disponibles dans le trésor mental de l'auditeur et prêts à être activés...

Ce que postule Saussure ici, c'est une suspension de l'écoute qui vaut suspension du texte, immobilisé dans un espace-temps sémiotique réversible, suspendu entre la temporalité de l'écoute et la spatialité de sa lettre. Et c'est ce compromis quasiment synesthésique entre le phonème et le graphème qui, au cœur même de la pensée saussurienne, pourrait être à l'origine de toute la spéculation anagrammatique. Nous employons le qualificatif de synesthésique à dessein car il n'est pas exclu que Saussure ait effectivement été synesthète : cet épisode peu connu de la biographie du linguiste genevois pourrait d'ailleurs avoir fortement déterminé sa conception quasiment syncrétique (à la fois orale et scripturale) du texte. Mais que Saussure ait vraiment été atteint de synesthésie ${ }^{17}$ ou non importe peu; ce qui compte c'est que, considérée comme une métaphore de sa quête scientifique sur l'anagramme, la démarche synesthésique est révélatrice d'un mode d'appréhension du texte et de la textualité tout à fait singulier. Si l'expérience synesthésique de Saussure - réelle ou imaginaire - est digne du plus grand intérêt, c'est pour tout ce qu'elle peut nous apprendre sur le principe de conversion son-lettre que l'on trouve constamment à l'œuvre dans le travail de déchiffrement de l'anagramme.

L'enquête menée en 1882 par Théodore Flournoy (1854-1921) et son collaborateur, disciple et cousin germain Édouard Claparède (1873-1940) sur les phénomènes d' " audition colorée ", à laquelle Saussure, dont les réponses font fortement penser à des phénomènes de synopsie, prit part en tant que sujet enquêté ${ }^{18}$, prélude à la contradiction fondamentale, définitivement irrésolue, car plusieurs fois soulevée mais jamais véritablement tranchée, de l'appréhension visuelle d'un phénomène que Saussure sait pourtant d'essence acoustique

15 Meschonnic Henri, Critique du rythme. Anthropologie historique du langage, Paris, Verdier, 1982, p. 299.

16 Baudrillard Jean, "L'anagramme ", L'échange symbolique et la mort, Paris, Gallimard, 1976, p. 285-308.

17 Mazzeo Marco, "Les voyelles colorées : Saussure et la synesthésie ", Cahiers Ferdinand de Saussure, $\mathrm{n}^{\circ}$ 57, 2004, p. 129-143.

18 Cifali Mireille, "Réponse à une enquête sur l'audition colorée ", Le Bloc-Notes de la psychanalyse, $\mathrm{n}^{\circ} 3,1983$, p. 111-131. 
et qui le conduit à constamment penser les sons comme des lettres ${ }^{19}$. On assiste en lisant l'enquête - passionnante - de Flournoy ${ }^{20}$ aux mêmes hésitations, aux mêmes atermoiements, aux mêmes irrésolutions qui présideront à la recherche autour de l'anagramme, que Saussure s'efforce de formaliser en termes phonétiques mais qu’il ne cessera de penser en termes alphabétiques. Synapse du son et de la lettre, le nouveau complexe formulé par Saussure sous forme d'algorithme, à savoir " l'être [voyelle/lettre] ${ }^{21}$ ", qu'il appellera plus tard le «gramme ", est le lieu où la vision rencontre l'audition ${ }^{22}$. Ce que nous

19 Dans le chapitre qu'il consacre aux photismes alphabétiques (audition colorée des voyelles et des consonnes), Théodore Flournoy transcrit un large extrait tiré d'une réponse au questionnaire qu'il juge particulièrement instructive à cet égard et qu'il dit devoir "à l'obligeance d'un éminent linguiste, M. X. ». En voici le début : " Je ne crois pas pouvoir répondre à la question (sur la couleur des voyelles) dans les termes où elle est posée. Car voici la circonstance qui me frappe : Nous écrivons en français la même voyelle de quatre manières différentes dans terrain, plein, matin, chien. Or quand cette voyelle est écrite ain, je la vois jaune pâle voyelle comme une brique mal cuite au four ; quand elle est écrite ein, elle me fait l'effet d'un réseau de veines violacées; quand elle est écrite in, je ne sais plus du tout quelle sensation de couleur elle évoque dans mon esprit, et je suis disposé à croire qu'elle n'en évoque aucune ; enfin si elle s'écrit en (ce qui n'arrive qu'après un $i$ précédent), l'ensemble du groupe ien me rappelle assez un enchevêtrement de cordes de chanvre encore fraîches, n'ayant pas encore pris la teinte blanchâtre de la corde usée " (Flournoy Théodor, Des phénomènes de synopsie (audition colorée) Photismes - schèmes visuels - personnifications, Paris-Genève, Alcan-Eggimann, 1893, p 50). Ce n'est que dans les années 80 qu'on apprendra, grâce à une note retrouvée par Mireille Cifali (op. cit.), que l'« éminent linguiste » dont Flournoy avait masqué l'identité sous le cryptonyme « $\mathrm{M}$. X. » n'est autre que son jeune confrère Ferdinand de Saussure, alors âgé de 35 ans. En effet, celui qui, quelques années plus tard, dans le cadre d'une enquête sur le phénomène glossolalique, serait amené à se pencher, à la demande de Théodore Flournoy (1900), sur le cas fascinant de la médium et spirite Catherine-Élise Müller, plus connue sous le nom d'Hélène Smith, pour expertiser son parler extatique, avait de fait déjà été sollicité par le psychologue suisse dans le cadre de son enquête sur le phénomène synesthésique (cf. Federico Bravo, "Écrire en langues. Un cas de glossolalie poétique : la jitanjáfora de Mariano Brull ", Pays de la langue. Pays de la poésie, Pau, Laboratoire de Recherches en Langues et Littératures Romanes-Éditions Covedi, 1998, p. 185-195).

20 Flournoy Théodor, Des phénomènes de synopsie (audition colorée) Photismes - schèmes visuels personnifications, Paris-Genève, Alcan-Eggimann, 1893.

21 Saussure représente cet "être " sous forme d'algorithme en plaçant à l'intérieur des crochets l'inscription "voyelle $x$ " au-dessus de "lettre $\mathrm{x}$ ». Une note de bas de page rédigée par Théodore Flournoy explique : "C'est-à-dire le tout complexe formé par la synthèse mentale des sons et des signes graphiques" (Flournoy, op. cit., p. 51). Voici le passage en question : "Ce n'est donc pas, semble-t-il, la voyelle comme telle, c'est-à-dire telle qu'elle existe pour l'oreille, qui appelle une certaine sensation visuelle correspondante. D'un autre côté, ce n'est pas non plus la vue d'une certaine lettre ou d'un certain groupe de lettres qui appelle cette sensation. Mais c'est la voyelle en tant que contenue dans cette expression graphique, c'est l'être imaginaire que forme cette première association d'idées qui, par une autre association, m’apparaît comme doué d'une certaine consistance et d'une certaine couleur, quelquefois aussi d'une certaine forme et d'une certaine odeur. Ces attributs de couleur et autres ne s'attachent pas, autrement dit, à des valeurs acoustiques, mais à des valeurs orthographiques, dont je fais involontairement des substances. L'être [voyelle $\mathrm{x} /$ lettre $\mathrm{x}$ ] est caractérisé par tel aspect, telle teinte, tel toucher " (Flournoy, 1893, p. 51).

22 Cf. Flournoy, op. cit., p. 51, et Federico Bravo, "L'écriture-signature : noms, anagrammes, cryptonymes ", La signature. Regards croisés autour d'une pratique sémiotique millénaire, F. Bravo (éd.), Presses Universitaires de Bordeaux, 2011, p. 131. 
apprend Saussure en dernière instance c'est que toute lecture est synesthésique ou, pour reprendre un vers célèbre de Quevedo, que lire c'est " écouter avec les yeux ${ }^{23} »$. De ce point de vue-là, ce n'est pas un moindre mérite de la pensée saussurienne que d'avoir imaginé une entité nouvelle - synthèse mentale des sons et des signes graphiques, annonciatrice du " gramme " postulé pour le Saturnien -, à l'interface de ces deux concepts mitoyens que sont le son et le signe graphique, l'activation de l'un ayant pour effet d'entraîner automatiquement - un peu comme le goût déclenche l'olfaction - l'activation de l'autre : l'anagramme qui fait tantôt appel aux propriétés graphiques du texte, tantôt à ses propriétés phoniques, opère le dépassement synesthésique de ce clivage. Le texte peut être oral, écrit ou encore gestuel, mais la textualité, elle, n'est ni l'un ni l'autre parce qu'elle est, pour ainsi dire, indépendante des matériaux discursifs mis en œuvre.

Suspensive, la lecture postulée par Saussure l'est à sa manière car, multivectorielle, elle aspire par le jeu des échos, des réminiscences sonores et des répétitions dont elle est le théâtre à rendre synchrones dans la mémoire visuelle ou acoustique de celui qui lit ou qui écoute le poème des séquences qui sont consécutives dans leur production : reconnaître un hypogramme dépecé dans le corps du texte poétique suppose en effet une désautomatisation de la lecture, ce qui revient à dire un réapprentissage de l'écoute désormais focalisée sur l'identification de signifiants onomastiques, ces noms propres dont la linéarité du texte brouille la reconnaissance et dont Saussure, en les plaçant au centre du dispositif, fait le moteur même de la lecture. Il n'est pas interdit alors de voir dans la lecture constamment sélective à laquelle Saussure soumet les textes, une projection littérale de la lecture flottante, desarrimée, suspensive, en égal suspens, en balance, infraconsciente, non focalisée, écoute en association libre que postule la psychanalyse. Mieux qu'une autre façon d'écrire ou de lire le texte, l'anagramme serait donc une autre façon de le faire parler, car comme le dit Alberto Manguel, « tout écrit dépend de la générosité de son lecteur ${ }^{24}$ ". Le texte que Saussure entend systématiser à travers l'anagramme englobe lui aussi deux instances : l'instance d'écriture et l'instance de lecture et engage, avec elles, leurs mémoires respectives.

Pour Saussure, avons-nous dit, le texte poétique est la paraphrase phonique d'un mot : le poème n'étant qu'un nom textualisé, le paradoxe se laisse ainsi manier en disant que le paragramme - nom épanoui en poème ${ }^{25}$ - opère la réduction de l'ensemble du texte à un nom propre, mais que ce texte est lui-même irréductible aux contextes qui accompagnent et son engendrement

23 Bravo Federico, "El saber del escritor: por una teoría de la cita ", Bulletin hispanique, 97, num. 1, janvier-juin 1995, p. 361-374.

24 Manguel Alberto, Une histoire de la lecture, Arles, Actes Sud, 1998.

25 Willemart Philippe, De l'inconscient en littérature, Liber, Montréal, 2008, p. 115. 
et sa réception. Les recherches de Saussure font apparaittre que le langage est structuré comme un diagramme (comme un réseau de connexions) et que l'anagramme n'est qu'une manifestation particulière de ce diagrammatisme. En effet, la théorie de l'anagramme repose, d'une part, sur l'idée de latence verbale (présence de structures latentes, en attente d'être réactivées) et d'autre part, sur le pouvoir combinatoire et diagrammatique du signe. Aussi, lorsqu'on examine toutes les possibilités théoriques que permet d'envisager le dispositif, on constate que le nom propre qui sert de base à l'hypogramme est, d'un point de vue purement théorique, soumis à deux séries de variables, d'ordre psychogénique les unes (soit le procédé est conscient, soit il échappe à la conscience du poète), d'ordre chronogénique les autres (soit la recherche du nom est antérieure à la composition du poème soit le nom en est une émanation). Si l'on considère le travail d'écriture comme un procès d'interaction langagière, le nom propre que Saussure situe à la source de la création poétique (ce que Julia Kristeva appelle le géno-texte ou l'engendrement de la formule ${ }^{26}$ ) n'est pas nécessairement un " avant " par rapport au texte, du moins peut-on en faire l'hypothèse ici : il est probablement un "pendant" voire un "après ". En effet, la chronologie de raison qui fait du nom propre le "pré-texte » du Saturnien se laisse renverser, pour peu qu'on tienne compte de la façon dont le discours agit ou interagit à son tour, à mesure qu'il se construit, avec l'instance de parole qui le porte. En d'autres termes : le nom propre peut, comme le prescrit Saussure, obéir à un projet onomastique initial en tant qu'hypotexte, mais il peut aussi prendre sa source dans la rencontre de cette instance agissante qu'on appelle l'auteur avec ce complexe signifiant qu'on appelle le texte en tant que co-texte ou qu'hypertexte. Le nom dont Saussure fait non seulement le fondement mais aussi le préalable de toute création pourrait donc ne pas s'imposer d'emblée, comme un donné, à l'esprit du poète, mais naître de la rencontre ou, pour reprendre le terme de Lacan, $\mathrm{du}$ " dialogue " du poète avec son texte. Ces deux variables génèrent ainsi six hypothèses de travail différentes : soit le nom est un avant-texte, soit il est un co-texte, soit un après-texte, et dans les trois cas de figure il peut obéir soit à un projet conscient soit à des mécanismes inconscients.

On le voit : entre le tout intentionnel et le tout inconscient, il pourrait y avoir une troisième voie dont Saussure lui-même a suggéré l'existence en affirmant que "la poésie comptait les phonèmes ${ }^{27}$ " : la poésie donc en tant qu'instance, non le poète en tant qu'individualité consciente. Comme la parole en général - mais cela excède de très loin le cadre de la seule activité langagière et peut se dire pratiquement de toute activité humaine -, l'écriture naît de la

26 Kristeva Julia, « Pour une sémiologie des paragrammes ", Recherches pour une sémanalyse, Paris, Seuil, 1969, p. 113-146.

27 Starobinski, ibid., p. 40. 
rencontre d'un projet et d'une mise en acte : elle est un compromis entre ce que je veux dire qui me fait dire quelque chose et ce que me fait dire à son tour ce que je dis. Cette interaction-là n'est pas plus le fruit du hasard qu'elle n'est l'émanation d'un projet " conscient ». Le dit est toujours à la fois contraint et contrainte.

À la lumière des questions examinées, je proposerai, en guise de conclusion, de lire l'anagramme de Saussure comme une métaphore de la textualité ou plutôt comme l'expression d'une autre textualité qui nous invite à appréhender dynamiquement le texte comme un passage et qui ferait voler en éclats les critères classiquement postulés de cohérence, de cohésion, de progression thématique et de séquentialité. À la cohérence (c'est-à-dire, à organisation logique de la pensée autour d'une intention globale), il substitue la logique du signifiant, un signifiant qui, pour reprendre l'expression de JeanClaude Chevalier, aurait ses raisons que le signifié ignore ${ }^{28}$. À la cohésion (qui repose crucialement sur l'intégration syntaxique des constituants textuels), il oppose un autre niveau d'articulation du texte, car en découvrant la présence de mots cachés sous les mots, Saussure met en lumière l'existence d'une syntaxe cachée sous la syntaxe, une vraie syntaxe des sons construite à l'image de celle qui, au niveau supérieur de l'analyse, gouverne les mots ou commande la phrase. À la progression thématique (qui veut que le texte se caractérise par l'articulation d'informations anciennes et d'informations nouvelles), il substitue celle de progression sémiotique, le texte n'étant autre chose que le produit de la contextualisation du nom propre qui a servi de modèle a sa construction. Enfin à la séquentialité, il oppose une tabularité ouverte et appuyée du texte, qui le décuple, le délinéarise et le rend finalement irréductible à la somme des cotextes dont il est porteur. Dans ce sens, le texte saussurien est un véritable observatoire de la textualité.

Federico Bravo

EA 4656 AMERIBER

Université Bordeaux Montaigne Federico.Bravo@u-bordeaux-montaigne.fr

28 Chevalier Jean-Claude, Michel Launay et Maurice Molho, "La raison du signifiant ", Modèles linguistiques, t. VI, fascicule 2, 1984, p. 27-41. 


\section{Résumé}

L'aptitude migratoire des unités textuelles dont se sustente le dispositif paragrammatique imaginé par Ferdinand de Saussure dans ses Cahiers d'anagrammes relève d'une conception tectonique du texte à la croisée de l'oral et de l'écrit. Délinéarisé, déconstruit, tabularisé, le texte devient surface mouvante, en trompe l'œil. À la fois contenu latent (hypotexte) et antécédent du texte (prétexte), l'anagramme invite à s'interroger sur l'articulation du binôme texte/ contexte, à réfléchir à l'irréductible du texte et à repenser la textualité comme une forme de dépassement du clivage oralité/scripturalité.

\section{Mots-clés}

Texte, cotexte, anagramme, Ferdinand de Saussure.

\section{Abstract}

The paradigmatic outflow which underpins the anagrammatic device imagined by Ferdinand de Saussure in his anagram notebooks shows a tectonic conception of text at the crossroads of oral and written. Delinearized, deconstructed, tabularised, the text becomes a moving discourse beneath the discourse. Both latent structure (hypotext) and antecedent of the text (pre-text), the anagram invites us to question the articulation between text and context, to reflect on the irreducibility of the text and to rethink textuality as a way of going beyond the traditional opposition between orality and scripturality.

\section{Keywords}

Text, cotext, anagram, Ferdinand de Saussure. 